\title{
Phase diagram of imbalanced fermions in optical lattices
}

\author{
Xiaoling Cui and Yupeng Wang \\ Beijing National Laboratory for Condensed Matter Physics and Institute of Physics, \\ Chinese Academy of Sciences, Beijing 100190, China
}

(Dated: March 5, 2022)

\begin{abstract}
The zero-temperature phase diagrams of imbalanced fermions in 3D optical lattices are investigated to evaluate the validity of the Fermi-Hubbard model. It is found that depending on the filling factor, $s$-wave scattering strength and lattice potential, the system may fall into the normal $(N)$ phase, magnetized superfluid $\left(\mathrm{SF}_{M}\right)$ or phase separation of $N$ and BCS state. By tuning these parameters, the superfluidity could be favorable by enhanced effective couplings or suppressed by the increased band gap. The phase profiles in the presence of a harmonic trap are also investigated under LDA, which show some exotic shell structures compared to those without the optical lattice.
\end{abstract}

In the past few years, great experimental progress has been achieved in studying ultracold Fermi gases with polarization $1,2,3,4,5$. With two unequal mixtures of cold ${ }^{6} \mathrm{Li}$ atoms in a harmonic trap ${ }^{1,2}$, a clear evidence of phase separation with an unpolarized superfluid (BCS) core and a normal $(N)$ shell around that has been observed in experiment. Theoretically $6,7,8,9,10,11,12,13$, many other ground state candidates have been proposed in such systems, including magnetized superfluid $\left(\mathrm{SF}_{M}\right)$, phase separation (PS), and Fulde-Ferrell-Larkin-Ovchinnikov (FFLO) state with finite momentum pairing by tuning the interaction parameter $1 / k_{F} a_{s}$, the polarization $P=\delta n / n$ or Zeeman field $h$. Since the optical lattice height $V_{0}$ is also tunable, it is very interesting to study its effect on the new phase diagram. For equal mixtures, a second-order quantum phase transition between superfluid (SF) and insulating (IN) phases has been addressed both experimentally at a critical lattice height $V_{c}$ at resonance ${ }^{14}$ and theoretically ${ }^{15,16}$ based on the secondorder perturbation theory. Besides, there are also works on imbalanced fermions in optical lattices focusing on $\mathrm{IN}^{17}, \mathrm{FFLO}^{18}$ and $\mathrm{SF}_{M}{ }^{19}$ phases, based on an effective Fermi-Hubbard model.

In this work, starting from the exact lattice spectrum, we study the ground state phase diagram of imbalanced two species Fermi gases trapped in 3D optical lattices, in terms of the total filling factor $n$, polarization $P, s$-wave scattering length $a_{s}$ and lattice potential $V_{0}$. Limited by the numerical attainment, the FFLO-type pairing is not considered. The total pairing reciprocal lattice momentums involved in our simulation are up to the six smallest non-zero ones, which turn out to be more and more important as $V_{0}$ increases. Sufficient multiple bands have been taken into account to ensure the accuracy especially in the strong coupling regime. We demonstrate that there are two contradictory effects of $V_{0}$ on the SF phase, depending on the average filling factor $n$. One is the enhanced density of states (DOS) inside each band which effectively increases the coupling strength and thus is favorable to SF; the other is the broadened band gap or discontinuity of DOS which is against SF. One key point is that besides tuning $a_{s}$ through the Feshbach resonance(FR), $V_{0}$ can also be tuned and drive the system from weak to strong coupling regime, provided that the filling factor is properly fixed. An obvious evidence is the emergence of $\mathrm{SF}_{M}$ phase for deep optical lattices at particular filling regimes, even in far BCS side of FR. We also propose that the critical polarization versus total filling factor diagram obtained can be used to evaluate the validity of the usual Fermi-Hubbard model. The phase profile in the presence of an external harmonic trap, which is more relevant to the practical experiment will be studied with local density approximation (LDA) finally. Some exotic structures appear, reflecting the uniqueness of the optical lattices.

In a recent experiment ${ }^{14}$, two hyperfine states of ultracold ${ }^{6} \mathrm{Li}$ atoms, $\left|F=1 / 2, m_{F}=1 / 2\right\rangle(|\uparrow\rangle)$ and $\left|F=1 / 2, m_{F}=-1 / 2\right\rangle(|\downarrow\rangle)$, had been successfully loaded to an optical lattice. The low-energy interactions are characterized by a single $s$-wave scattering length $a_{s}$, which can be tuned by FR. Such a system can be well described by the one-channel Hamiltonian

$$
\begin{aligned}
H= & \int d \mathbf{r} \sum_{\sigma=\uparrow, \downarrow} \psi_{\sigma}^{\dagger}(\mathbf{r}) \hat{H}_{0}(\mathbf{r}) \psi_{\sigma}(\mathbf{r})+ \\
& g \int d \mathbf{r} \psi_{\uparrow}^{\dagger}(\mathbf{r}) \psi_{\downarrow}^{\dagger}(\mathbf{r}) \psi_{\downarrow}(\mathbf{r}) \psi_{\uparrow}(\mathbf{r}),
\end{aligned}
$$

where $\hat{H}_{0}=\sum_{i=x, y, z}-\hbar^{2} \partial_{i}^{2} / 2 M+V_{0} \sin ^{2}\left(\pi x_{i} / a\right) ; a=$ $\lambda / 2$ is the period of the lattice generated in each direction by two oppositely propagating lasers with wavelength $\lambda$; $V_{0}$ is the lattice height which is usually measured by the recoil energy $E_{R}=\frac{\hbar^{2} \pi^{2}}{2 M a^{2}} ; g$ is the renormalized contact interaction constant between two species by eliminating the unphysical divergence due to the high-momentum contribution for fermi gases, $\frac{1}{g}=\frac{m}{4 \pi \hbar^{2} a_{s}}-\frac{1}{V} \sum_{q} \frac{1}{2 \epsilon_{q}}$.

In the framework of mean-field approach, we expand first each field operator in terms of eigenwave functions of $\hat{H}_{0}, \psi_{\sigma}(\mathbf{r})=\sum_{\mathbf{n k}} \phi_{\mathbf{n k}}(\mathbf{r}) \psi_{\mathbf{n k} \sigma}$. The Bloch wave functions $\phi_{\mathbf{n k}}(\mathbf{r})=\frac{1}{\sqrt{V}} \sum_{\mathbf{G}} a_{\mathbf{n k}}(\mathbf{G}) e^{i(\mathbf{k}+\mathbf{G}) \cdot \mathbf{r}}$ and energies $\epsilon_{\mathbf{n k}}$ are obtained from the Schrödinger equation

$$
\begin{gathered}
\sum_{\mathbf{G}^{\prime}}\left\{\left[\frac{\hbar^{2}}{2 M}(\mathbf{k}+\mathbf{G})^{2}+\frac{3 V_{0}}{2}\right] \delta_{\mathbf{G G}^{\prime}}-\frac{V_{0}}{4} \sum_{i} \delta_{\mathbf{G} \pm \frac{2 \pi}{a} \mathbf{e}_{i}, \mathbf{G}^{\prime}}\right\} \\
a_{\mathbf{n k}}\left(\mathbf{G}^{\prime}\right)=\epsilon_{\mathbf{n k}} a_{\mathbf{n k}}(\mathbf{G}),
\end{gathered}
$$


where $\mathbf{n}=\left\{n_{x}, n_{y}, n_{z}\right\}=s, p, \ldots$ indicate the band indices; $\mathbf{k}$ lie in the first Brillouin zone (BZ) and $\mathbf{G}=2 \pi / a\left(l_{x}, l_{y}, l_{z}\right)$ is the reciprocal lattice vector. The solutions satisfy $\sum_{\mathbf{G}} a_{\mathbf{n k}}^{*}(\mathbf{G}) a_{\mathbf{n}^{\prime} \mathbf{k}}(\mathbf{G})=\delta_{\mathbf{n n}^{\prime}}$ and $a_{\mathbf{n},-\mathbf{k}}(-\mathbf{G})=a_{\mathbf{n k}}^{*}(\mathbf{G})$. The standard mean-field treatment gives

$$
\begin{aligned}
H- & \sum_{\sigma} \mu_{\sigma} N_{\sigma}=\sum_{\mathbf{n k} \sigma}\left(\epsilon_{\mathbf{n k}}-\mu_{\sigma}\right) \psi_{\mathbf{n k} \sigma}^{\dagger} \psi_{\mathbf{n k} \sigma}- \\
& \sum_{\mathbf{m n k}}\left(\Delta_{\mathbf{m n k}}^{*} \psi_{\mathbf{m}-\mathbf{k} \downarrow} \psi_{\mathbf{n k} \uparrow}+\text { h.c. }\right)-\frac{V}{g} \sum_{\mathbf{Q}}\left|\Delta_{\mathbf{Q}}\right|^{2}(3)
\end{aligned}
$$

with

$$
\begin{aligned}
\Delta_{\mathbf{Q}} & =-\frac{g}{V} \sum_{\mathbf{m n k}} M_{\mathbf{m n k}}^{\mathbf{Q}}\left\langle\psi_{\mathbf{m}-\mathbf{k} \downarrow} \psi_{\mathbf{n k} \uparrow}\right\rangle, \\
\Delta_{\mathbf{m n k}} & =\sum_{\mathbf{Q}} \Delta_{\mathbf{Q}} M_{\mathbf{m n k}}^{\mathbf{Q} *},
\end{aligned}
$$

and $M_{\mathbf{m n k}}^{\mathbf{Q}}=\sum_{\mathbf{G}} a_{\mathbf{m}-\mathbf{k}}(-\mathbf{G}) a_{\mathbf{n k}}(\mathbf{G}+\mathbf{Q})$. Since $M_{\mathbf{m n k}}^{\mathbf{Q}=0}=\delta_{\mathbf{m n}}$ and if $m \neq n M_{\mathbf{m n k}}^{\mathbf{Q} \neq 0}$ are quite small, in the following text we only consider pairing within each single band, which means $\Delta_{\mathbf{m n k}} \approx \Delta_{\mathbf{n k}} \delta_{\mathbf{m n}}$. In such a case, the Hamiltonian can be easily diagonalized, and the thermodynamic potential is calculated at $T=0$ as

$$
\begin{aligned}
\frac{\Omega}{V}= & \frac{1}{V} \sum_{\mathbf{n k}}\left\{\Theta\left(-E_{\mathbf{n k}+}\right) E_{\mathbf{n k}+}+\Theta\left(-E_{\mathbf{n k}-}\right) E_{\mathbf{n k}-}+\right. \\
& \left.\epsilon_{\mathbf{n k}}-\mu-\sqrt{\left(\epsilon_{\mathbf{n k}}-\mu\right)^{2}+\Delta_{\mathbf{n k}}^{2}}\right\}-\sum_{\mathbf{Q}} \frac{\left|\Delta_{\mathbf{Q}}\right|^{2}}{g},(5)
\end{aligned}
$$

with $E_{\mathbf{n k} \pm}=\sqrt{\left(\epsilon_{\mathbf{n k}}-\mu\right)^{2}+\Delta_{\mathbf{n k}}^{2}} \mp h$ where $\mu=\left(\mu_{\uparrow}+\right.$ $\left.\mu_{\downarrow}\right) / 2$ and $h=\left(\mu_{\uparrow}-\mu_{\downarrow}\right) / 2$. From $\partial \Omega / \partial \Delta_{\mathbf{Q}}^{*}=0$ and $N_{\sigma}=-\partial \Omega / \partial \mu_{\sigma}$, we get the gap and density equations as

$$
\begin{aligned}
-\frac{\Delta_{\mathbf{Q}}}{g} & =\frac{1}{V} \sum_{E_{\mathbf{n k} \pm>0}} \frac{M_{\mathbf{n k}}^{\mathbf{Q}} \Delta_{\mathbf{n k}}}{2 \sqrt{\left(\epsilon_{\mathbf{n k}}-\mu\right)^{2}+\Delta_{\mathbf{n k}}^{2}}} \\
n & =\frac{1}{N_{L}}\left(\sum_{\mathbf{n k}} 1-\sum_{E_{\mathbf{n k} \pm}>0} \frac{\epsilon_{\mathbf{n k}}-\mu}{\sqrt{\left(\epsilon_{\mathbf{n k}}-\mu\right)^{2}+\Delta_{\mathbf{n k}}^{2}}}\right) \\
\delta n & =\frac{1}{N_{L}}\left(\sum_{E_{\mathbf{n k}+}<0} 1-\sum_{E_{\mathbf{n k}-<0}} 1\right) .
\end{aligned}
$$

Here $N_{L}$ is the total number of lattice sites, $n=\left(N_{\uparrow}+\right.$ $\left.N_{\downarrow}\right) / N_{L}$ and $\delta n=\left(N_{\uparrow}-N_{\downarrow}\right) / N_{L}$ are the total filling factor and the difference, respectively. Hereafter we scale all the energies in unit of $E_{R}$ and the momenta of $2 \pi / a$.

To make the numerical simulations attainable but still retain the essence of the problem, besides $\mathbf{Q}=0$ we consider other six non-zero $\mathbf{Q}$ : $( \pm 1,0,0),(0, \pm 1,0),(0,0, \pm 1)$. Due to $M_{\mathbf{n k}}^{\mathbf{Q}}=M_{\mathbf{n} \pm \mathbf{k}}^{ \pm \mathbf{Q}}$ and the isotropy of $3 \mathrm{D}$ cubic lattices, all six non-zero $\mathbf{Q}$ share the same pairing amplitude $\Delta_{1}$. Therefore we get two coupled gap equations in terms of $\Delta_{0}$ and $\Delta_{1}$. For a realistic numerical simulation, we apply a cutoff momentum
$\left|q_{\Lambda}\right|=\frac{3}{2}(1,1,1)$ in the renormalization and correspondingly consider the lowest three bands $(s, p, d)$ in each direction of lattice spectrum. This truncation allows totally $n=54$ atoms per site at most, which is well above the experimental interest as well as ours in this paper.

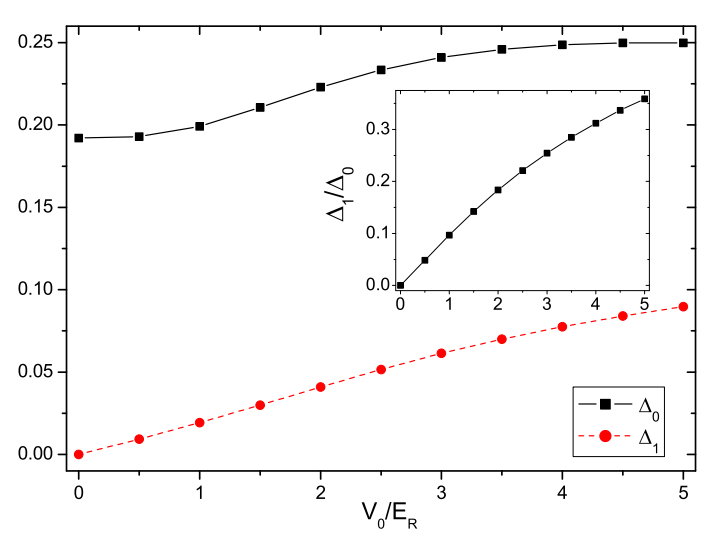

FIG. 1: (color online) $\Delta_{0}, \Delta_{1}$ and $\Delta_{1} / \Delta_{0}$ (see inset) vs lattice potentials $V_{0}$ for equal mixtures. $a / a_{s}=-3$. The averaged filling is fixed to be $n=1$.

Before turning to the phase diagram of imbalanced system, first we analyze the necessity of involving nonzero $\mathbf{Q}$ in gap equations for equal mixtures. Fig. 1 shows $\Delta_{0}, \Delta_{1}$ and their ratio as a function of lattice potential $V_{0}$ at half filling $n=1$. It is shown that the $\mathbf{Q} \neq 0$ pairing becomes more and more important as $V_{0}$ increases. This effect can be understood as follows. Taking a very shallow 1D lattice for example, non-zero $M_{n k}^{Q}$ with $|Q|=2,4,6 \ldots$ and $1,3,5 \ldots$ only exist around kinetic energy-degenerate points $k=0$ and $k= \pm 1 / 2$ respectively, which contribute little to gap-equations and therefore produce a negligible $\Delta_{1}$. In the limit of $V_{0}=0$, these non-zero $M_{n k}^{Q}$ exactly cancel with each other in gap equations and finally only $Q=0$ pairing survives. However as $V_{0}$ increases, the eigen-vector $a_{n k}(G)$ evolves such that the area of non-zero $M_{n k}^{Q}$ expands from three discrete points in first BZ to considerable regions around them, leading to an increasing $\Delta_{Q}$ with $Q \neq 0$. Since our interest is still within $s$-band, the $|Q|=1$ pairings take a leading role among all non-zero ones, which is verified both numerically and analytically from a perturbation theory for shallow lattices. This is why we just take into account six smallest non-zero $\mathbf{Q}$ in $3 D$ case for not-sodeep lattices. The consideration of non-zero Q-pairing would produce a much stronger superfluidity especially for deep lattices, which can also be seen from the comparison of the previous two works ${ }^{20}$.

The ground state phase diagram in Fig. 2 is determined as follows. We first compare $\Omega_{B C S}(\mu, h=$ $\left.E_{\text {min }}, \Delta=\left\{\Delta_{0}, \Delta_{1}\right\}\right)$ with with $\Omega_{N}\left(\mu, h=E_{m i n}, \Delta=\right.$ $\{0\})$, with $\Delta_{0 / 1}$ obtained for unpolarized BCS state and $E_{\text {min }}=\operatorname{Min}_{n k}\left(\sqrt{\left(\epsilon_{n k}-\mu\right)^{2}+\Delta_{n k}^{2}}\right)$ its lowest excitation 
energy. If $\Omega_{B C S}>\Omega_{N}$ then the first-order phase transition point $h_{c}\left(<E_{\text {min }}\right)$ is given by

$$
\begin{aligned}
\Omega_{B C S}\left(\mu, h_{c}, \Delta=\right. & \left.\left\{\Delta_{0}, \Delta_{1}\right\}\right)=\Omega_{N}\left(\mu, h_{c}, \Delta=\{0\}\right),(8) \\
& n_{N}\left(\mu, h_{c}\right)=n .
\end{aligned}
$$

$P_{c}=\delta n_{N}\left(\mu, h_{c}\right) / n$ represents a critical point when PS is entirely composed by $N$ phase. Note that in this case the polarized SF or Sarma phase ${ }^{6}$ is unstable due to the negative superfluid density $\stackrel{11}{ }$. If $\Omega_{B C S}<\Omega_{N}$ then there should be a stable $\mathrm{SF}_{M}$ interpolating between BCS and $N$ phase. In free space at the $\mathrm{SF}_{M}$-N second-order transition point, $N$ denotes a fully polarized normal state with $P_{c}=1^{13}$. Correspondingly in optical lattices, we obtain $P_{c}=1$ at $n \ll 1$ and $|n-2| / n$ at $n \sim 2$, as shown by red solid circles in Fig. 2,

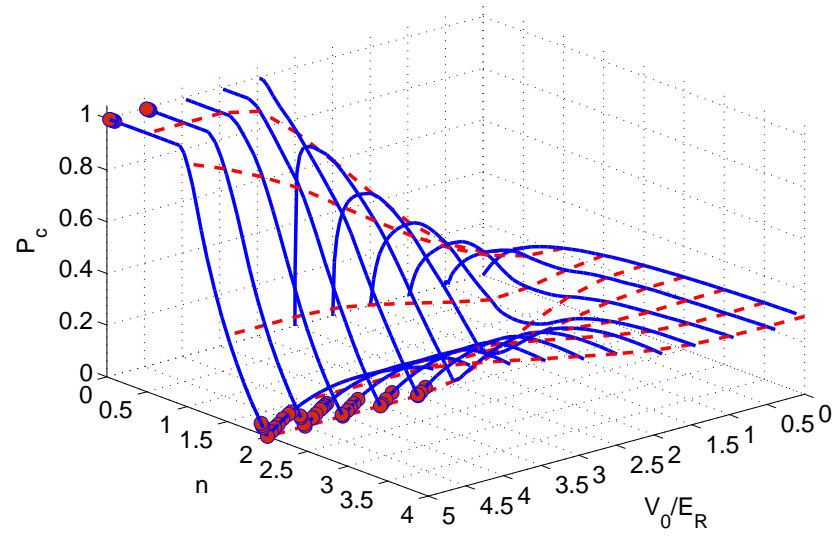

FIG. 2: (color online) Zero temperature phase diagram as a function of polarization $P_{c}=\delta n / n$, total filling factor $n$ and lattice height $V_{0} . \quad a / a_{s}=-3$. The red dashed(blue solid) lines show $P_{c}$ evolves with $V_{0}(n)$ for fixed $n\left(V_{0}\right)$. All the lines above denote the $P S-N$ boundaries, expect for red solid circles separating $\mathrm{SF}_{M}$ and $N$ phase instead.

We analyze that $P_{c}-V_{0}$ curves reveal two contradictory effects of increasing $V_{0}$ to SF depending on the filling factor $n$. As shown in Fig. 3, for $n \leq 1$ increasing $V_{0}$ will flatten each band and enhance DOS(almost inversely proportional to the band width); while at $n \sim 2$, increasing $V_{0}$ produces an entirely opposite effect due to the enlarged band gap. According to the standard BCS theory, the DOS at the Fermi surface dramatically affects the strength of $\mathrm{SF}$, as is also reflected by such contradictory effects. When $V_{0}=3 E_{R}, P_{c}$ increases to unity at small $n$ but reduces to zero at $n=2$, denoting the IN phase with $n_{\uparrow}=n_{\downarrow}=1$. For $n \in(1,2), P_{c}$ initially drops down and then goes up, indicating the competition between the above these two effects. Here the lattice enhancement of $P_{c}$ at $n \leq 1$ is similar to the enhancement of $T_{c}$ for equal mixtures in weak coupling limit ${ }^{21,22}$.

Next we turn to $P_{c}-n$ diagram for fixed $V_{0}$. As is well known in free space, a first-order BCS to $N$ phase transition takes place in weak coupling limit at $h_{c}=\frac{\Delta_{0}}{\sqrt{2}}$ and $P_{c}=\frac{3 h_{c}}{2 E_{F}}$, with the gap amplitude

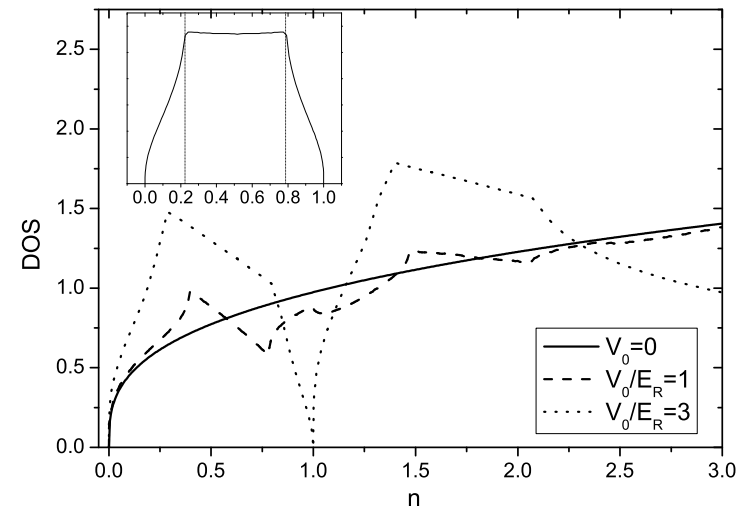

FIG. 3: Density of state(DOS) at the Fermi surface versus filling factor $n$ for single-spin atoms in 3D free space and in lattices with $V_{0} / E_{R}=1$ (no band gap) and $V_{0} / E_{R}=3$ (with band gap). Inset is DOS for non-interacting Hubbard model. The dotted lines therein denote two peaks of DOS at $(\mu=$ $\left.t, n_{p}=0.213\right)$ and $\left(3 t-\mu, 1-n_{p}\right)$.

$\Delta_{0}=\frac{8}{e^{2}} E_{F} \exp \left(-\frac{\pi}{2 k_{F}\left|a_{s}\right|}\right)$ and the interaction parameter $\eta=\frac{1}{k_{F} a_{s}}=\frac{a}{a_{s}}\left(3 \pi^{2} n\right)^{-\frac{1}{3}}$. $P_{c}$ will increase with $n$ all along from weak coupling $\operatorname{limit}(\eta \rightarrow-\infty)$ to the unitary $\operatorname{limit}(\eta \rightarrow 0)$. Within an optical lattice, however, the $P_{c}-n$ curve would be dramatically modified. In weak coupling limit with small $V_{0}$, the curve basically follows as that of DOS in Fig. 3, with a dip at $n_{d} \sim 2$ and correspondingly a peak at $n_{p}$. As $V_{0}$ increases, $n_{p}$ gradually moves to the left and finally vanishes at $n_{p}=0$, and finally $\mathrm{SF}_{M}$ state emerges at $n \ll 1$ or $n \sim 2$. Different from the $\mathrm{SF}_{M}$ studied by DMFT method under tight-binding model 19 , the phase shown here is purely due to the enhanced effective coupling by lattices. In this limit, two fermions are likely to form a molecule, and the BCS equation directly reduces to a Schrodinger equation for a single bound pair ${ }^{21,23}$. It is expected that as $V_{0}$ increases, the $\mathrm{SF}_{M}$ phase would extend to a larger or even the whole density region. Actually, the physics at $n \ll 1$ and $n \sim 2$ can be related to each other via particle-hole symmetry. The symmetry is essentially obvious within the background of Fermi-Hubbard model, $\epsilon_{\mathbf{k}}=0.5 t \sum_{i}\left(1-\cos \left(k_{i} a\right)\right)$. Since $(n, \mu)$ and $(2-n, 3 t-\mu)$ share the same $\{\Delta, h, \delta n, \Omega\}$ and thus the same critical $\delta n_{c}$, the critical polarization $P_{c}(n)$ for $n \leq 8$ follows as

$$
P_{c}=\left\{\begin{array}{cc}
1, & n \leq 1 \\
\frac{|n-2|}{\frac{n-n}{n}}, & 1<n \leq 5 \\
\frac{5}{n}, n \leq 8
\end{array}\right.
$$

We also compute the phase diagram at other $s$-wave couplings with fixed $V_{0}$ as shown in Fig. 4 Different from Fig. 2] it shows that the increasing $a / a_{s}$ always enhance SF and improve $P_{c}$ regardless of filling factors. At sufficiently strong coupling close to unitary, the particlehole symmetry in each band breaks down since it is en- 
ergetically favorable for particles in s-band to overcome the band-gap and form cooper pairs even at $n=2$. In this case the multi-band effect should be taken into account. This is why $\mathrm{SF}_{M}$ only turns up at $n \ll 1$ but not at $n \sim 2$ in unitary limit, shown as blue circles in Fig. 4 .

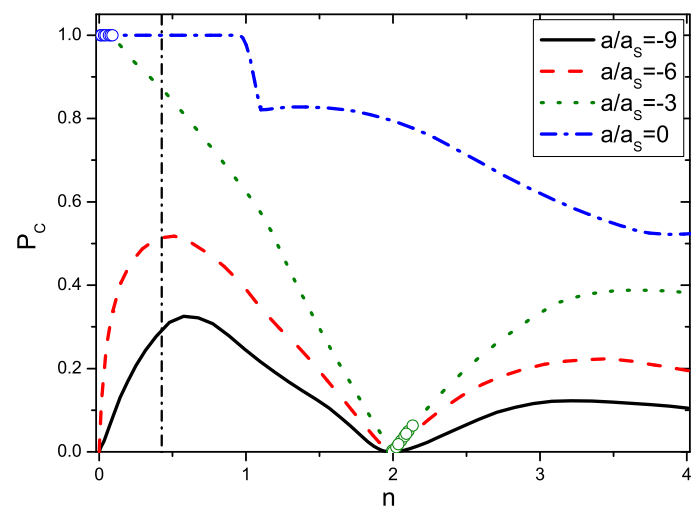

FIG. 4: (color online) $P_{c}$ versus $n$ diagram at different couplings. $V_{0}=3 E_{R}$. All lines denote $P S-N$ boundaries, except that the green and blue circles show $S F_{M}-N$ boundaries. The black dashed-dot line marks the upper limit of peak position based on Fermi-Hubbard model(see text).

We emphasize that the $P_{c}-n$ diagram in weak coupling limit can be used to evaluate the validity of tightbinding approximation(TBA) usually applied to the lattices. For Hubbard model, the DOS shows two peaks symmetric around half filling(see inset of Fig. 3), due to the van Hove singularity at $(\mu=t, n=0.213)$ and $(\mu=2 t, n=0.787)$. We also verified numerically that the peak position of $P_{c}$ at different couplings is never greater than 0.426 for arbitrary interactions $|U| / t$, twice as that for the first peak in DOS. This universal prop- erty could be used to justify the validity of TBA to realistic lattices. Apparently from Fig. 4 we see that the TBA is not applicable to $V_{0}=3 E_{R}$, since at really weak $\operatorname{coupling}\left(a / a_{s}=-9\right)$ the peak position $n_{p} \simeq 0.6>0.426$. The disagreement here indicates the deviation of the two lattice spectrum, and thus the necessity of adopting exact lattice spectrum for not-so-deep lattices.

Finally, it is also useful to consider the phase profile relevant to realistic experiments with an external harmonic potential $V(\mathbf{r})$. Under LDA, the system is assumed to be locally homogeneous with an averaged chemical potential $\mu(\mathbf{r})=\left(\mu_{0 \uparrow}+\mu_{0 \downarrow}\right) / 2-V(\mathbf{r})$ and position-independent difference $h=\left(\mu_{0 \uparrow}-\mu_{0 \downarrow}\right) / 2$. The phase at position $\mathbf{r}$ is determined by the local $(\mu(\mathbf{r}), h)$, which is also selfconsistently related to the total particle numbers, $s$-wave interaction and the lattice potential. Here we give several typical phase profiles with the filling factor in trap center larger than 2. For relatively shallow lattices and very weak $s$-wave interactions, a typical one from the trap center to the edge is: BCS-PN-IN-PN-BCS-PNFN (PN/FN: partially/fully polarized Normal). Starting from this profile, if $V_{0}$ increases, $\mathrm{PN}$ is very likely to be replaced by $\mathrm{SF}_{M}$ at positions where $n(\mathbf{r}) \sim 2$ or $n(\mathbf{r}) \ll 1$, while IN still survive in a certain region; But if $s$-wave interaction increases, then IN shrinks gradually, two BCS regimes merge together and $\mathrm{PN}$ gives rise to $\mathrm{SF}_{M}$, with only three phases left finally: $\mathrm{BCS}_{-} \mathrm{SF}_{M}-\mathrm{FN}$. In the latter case, much higher bands with continuous spectrum would be occupied, which makes the situation very similar to free space and the lattice effect is not obvious.

We thank Fei Zhou and An-Chun Ji for beneficial discussions. X.L.C. would like to thank the hospitality of UBC during her stay in Canada, where this work was initially started. This work is in part supported by NSFC under Grant No. 10574150 and 973-Project(China) under Grant No. 2006CB921300.
1 M. W. Zwierlein, A. Schirotzek, C. H. Schunck, W. Ketterle, Science 311, 492 (2006).

2 G. B. Partridge, W. Li, R. I. Kamar, Y. Liao, R. G. Hulet, Science 311, 503 (2006).

3 M. W. Zwierlein, C. H. Schunck, A. Schirotzek, W. Ketterle, Nature 442, 54 (2006).

${ }^{4}$ C. H. Schunck, Y. Shin, A. Schirotzek, M. W. Zwierlein, W. Ketterle, Science 316, 867 (2007).

5 Y.Shin, C. H. Schunck, A. Schirotzek, W. Ketterle, Nature 451, 689 (2008).

${ }^{6}$ G. Sarma, J. Phys. Chem. Solids 24, 1029 (1963).

7 P. Fulde, R. A. Ferrell, Phys. Rev. 135, A550 (1964); A. I. Larkin, Y. N. Ovchinnikov, Sov, Phys. JETP 20, 762 (1965).

8 P. F. Bedaque, H. Caldas, G. Rupak, Phys. Rev. Lett. 91, 247002 (2003).

9 D. E. Sheehy and L. Radzihovsky, Phys. Rev. Lett. 96, 060401 (2006).
10 Z. C. Gu, G. Warner and F. Zhou, cond-mat/0603091 (2006).

11 C. H. Pao, S. T. Wu and S. K. Yip, Phys. Rev. B. 73, 132506 (2006).

12 H. Hu and X. J. Liu, Phys. Rev. A. 73, 051603(R) (2006).

13 M. M. Parish, F. M. Marchetti, A. Lamacraft and B. D. Simons, Nature Physics 3, 124 (2007).

14 J. K. Chin, D. E. Miller, Y. Liu, C. A. Stan, W. Setiawan, C. Sanner, K. Xu and W. Ketterle, Nature 443, 961 (2006).

15 H. Zhai and T. L. Ho, Phys. Rev. Lett. 99, 100402 (2007).

16 E. G. Moon, P. Nikolić, and S. Sachdev, Phys. Rev. Lett. 99, 230403 (2007).

17 M. Iskin and C.A.R. Sá de Melo, Phys. Rev. Lett. 99, 080403 (2007).

18 T.K. Koponen, T. Paananen, J.-P. Martikainen, P. Torma, Phys. Rev. Lett. 99, 120403 (2007); T.K. Koponen, T. Paananen, J.-P. Martikainen, M.R. Bakhtiari, P. Torma, New J. Phys. 10, 045014 (2008). 
19 T.-L. Dao, M. Ferrero, A. Georges, M. Capone and O. Parcollet, Phys. Rev. Lett. 101, 236405 (2008).

20 Comparing two works on $S F-I N$ transition for equal mixtures at resonance, the critical lattice potential $V_{c}$ calculated in one $\mathrm{e}^{16}$ is almost one order of magnitude larger than in the other ${ }^{15}$, due to the consideration of couplings between different reciprocal lattice vectors.
21 R. Micnas, J. Ranninger, S. Robaszkiewicz, Rev. Mod. Phys. 62, 113 (1990).

${ }^{22}$ W. Hofstetter, J. I. Cirac, P. Zoller, E. Demler and M. D. Lukin, Phys. Rev. Lett. 89, 220407 (2002)

23 P. Nozières and S. Schmitt-Rink, J. Low Temp. Phys. 59, 195 (1985). 